\title{
Inflammasomes: Molecular Regulation and Implications for Metabolic and Cognitive Diseases
}

\begin{abstract}
Alexander J.S. Choi ${ }^{1}$, and Stefan W. Ryter ${ }^{2,3, *}$
Inflammasomes are specialized signaling platforms critical for the regulation of innate immune and inflammatory responses. Various NLR family members (i.e., NLRP1, NLRP3, and IPAF) as well as the PYHIN family member AIM2 can form inflammasome complexes. These multiprotein complexes activate inflammatory caspases (i.e., caspase-1) which in turn catalyze the maturation of select pro-inflammatory cytokines, including interleukin (IL)-1 $\beta$ and IL-18. Activation of the NLRP3 inflammasome typically requires two initiating signals. Toll-like receptor (TLR) and NOD-like receptor (NLR) agonists activate the transcription of pro-inflammatory cytokine genes through an NF-kBdependent priming signal. Following exposure to extracellular ATP, stimulation of the P2X purinoreceptor-7 (P2X $\left.X_{7} R\right)$, which results in $\mathrm{K}^{+}$efflux, is required as a second signal for NLRP3 inflammasome formation. Alternative models for NLRP3 activation involve lysosomal destabilization and phagocytic NADPH oxidase and /or mitochondria-dependent reactive oxygen species (ROS) production. In this review we examine regulatory mechanisms that activate the NLRP3 inflammasome pathway. Furthermore, we discuss the potential roles of NLRP3 in metabolic and cognitive diseases, including obesity, type 2 diabetes mellitus, Alzheimer's disease, and major depressive disorder. Novel therapeutics involving inflammasome activation may result in possible clinical applications in the near future.
\end{abstract}

\section{INTRODUCTION}

Inflammation involves a tightly regulated process, characterized by two complementary subsystems: the innate immune system

${ }^{1}$ Center for Sleep Medicine, Department of Pulmonary, Critical Care, and Sleep Medicine, Tufts University School of Medicine, ${ }^{2} J o a n$ and Sanford I. Weill Department of Medicine, New York-Presbyterian Hospital, Weill Cornell Medical College, New York, New York, USA, ${ }^{3}$ Division of Pulmonary and Critical Care Medicine, Brigham and Women's Hospital, Harvard Medical School, Boston, Massachusetts, USA

*Correspondence: str2020@med.cornell.edu

Received 28 April, 2014; accepted 1 May, 2014; published online 19 May, 2014

Keywords: cognitive disease, diabetes, inflammasome, inflammation, metabolism, obesity and the subsequent, highly adaptive immune system. The innate immune system is activated by invasive pathogens that target pattern recognition receptors (PRRs), such as membrane-bound Toll-like receptors (TLRs) and cytosolic NOD-like receptors (NLRs). While TLRs detect both extracellular and intracellular stimuli, NLRs respond to cytosolic agonists. The NLR family contains various proteins that act as scaffolds that can oligomerize into larger protein complexes, thereby inducing the formation of a molecular platform called the inflammasome. These multi-protein complexes contribute to the activation of inflammatory caspases (i.e., caspase-1) that result in the proteolytic processing and secretion of cytokines, including interleukin (IL)-1 $\beta$ and IL-18 (Martinon et al., 2009). Therefore, inflammasomes, which include members of the NLR family (i.e., NLRP1, NLRP3, and IPAF) as well as the PYHIN family member AIM2, represent critical mediators of cellular stress in the innate and adaptive immune systems (Fig. 1). This review will discuss the importance of inflammasome pathways, specifically, that of the most fully characterized NLRP3 inflammasome, in metabolic and cognitive diseases, including obesity, type 2 diabetes mellitus, Alzheimer's disease, and major depressive disorder (Fig. 2).

\section{STRUCTURAL ORGANIZATION}

\section{The NLR family}

The NLR protein family consists of the NLRPs (NLRP1-14, or NALP1-14) and the ICE-protease-activating factor (IPAF) subfamily. Most NLRs contain the nucleotide-binding and oligomerization (NACHT) domain, which enables activation of the proinflammatory cytokine signaling pathways via ATP-dependent oligomerization. The NACHT domain is usually surrounded by C-terminal leucine-rich repeats (LRRs) and an N-terminal caspase and recruitment domain (CARD) or pyrin domain (PYD) (Schroder and Tschopp, 2010). The PYD domain of NLRPs recruits the adaptor apoptosis-associated speck-like protein containing a caspase recruitment domain (ASC), which contains an N-terminal PYD and a C-terminal CARD. Furthermore, the CARD domain of ASC represents an essential component for inflammasome formation via caspase-1 binding (Mariathasan et al., 2004; Martinon et al., 2002).

The NLRP1, NLRP3, and IPAF inflammasomes harbor structural distinctions that enable caspase-1 recruiting. The NLRP1 inflammasome contains a C-terminal CARD that recruits procaspase-1 (Faustin et al., 2007) or pro-caspase-5 (Martinon et al., 2002). As a result, NLRP1 does not require recruitment of 


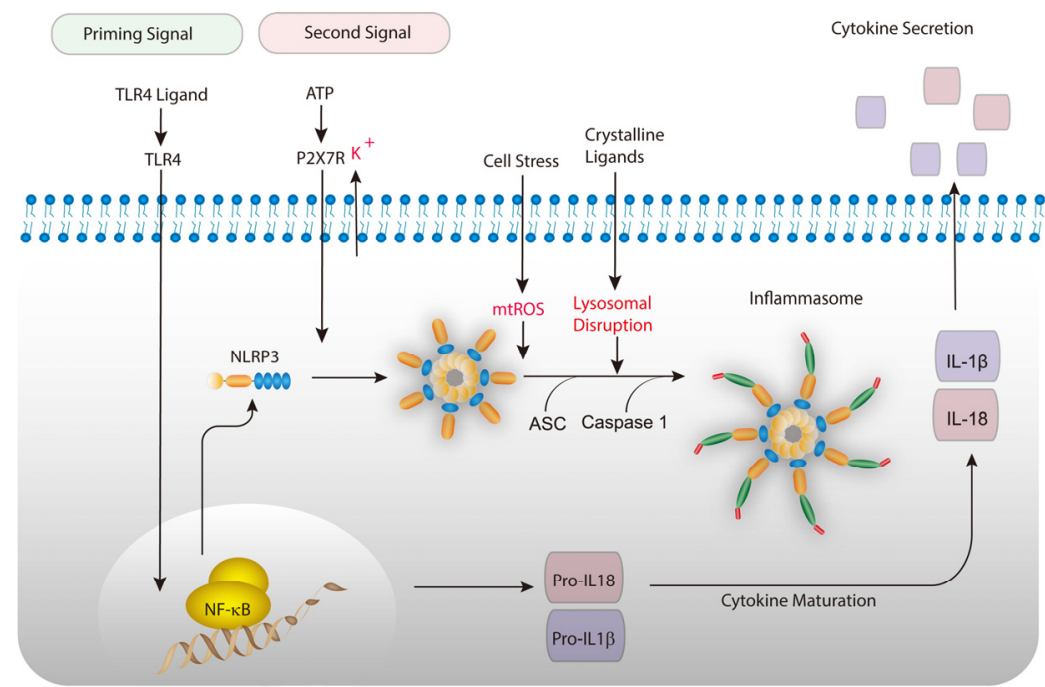

Fig. 1. The NLRP3 inflammasome regulates the caspase-1 dependent maturation and secretion of pro-inflammatory cytokines. Activation of the NLRP3 inflammasome typically requires a bimodal signaling pathway. A Toll-like receptor (TLR)dependent priming step activates the NF-кBdependent transcription of NLRP3 and the proforms of the pro-inflammatory cytokines (i.e., IL-1 $\beta$ and IL-18). A second signal involves the activation of the $\mathrm{P} 2 \mathrm{X}_{7} \mathrm{R}$ receptor by stimulation with exogenous ATP, which triggers potassium ion $\left(\mathrm{K}^{+}\right)$efflux. Oligomerization of NLRP3 is followed by recruitment of the adaptor molecule ASC and the proform of caspase-1, leading to the activation (cleavage) of caspase-1. Activated caspase-1 in turn catalyzes the cleavage of IL-1 $\beta$ and IL-18. The NLRP3 inflammsome may be activated by agents that cause mitochondrial dysfunction, leading to the generation of mitochondrial reactive oxygen species (ROS). Furthermore, the NLRP3 inflammasome may be activated by particulates such as monosodium urate or silica, in a mechanism that may involve disruption of the lysosomes.

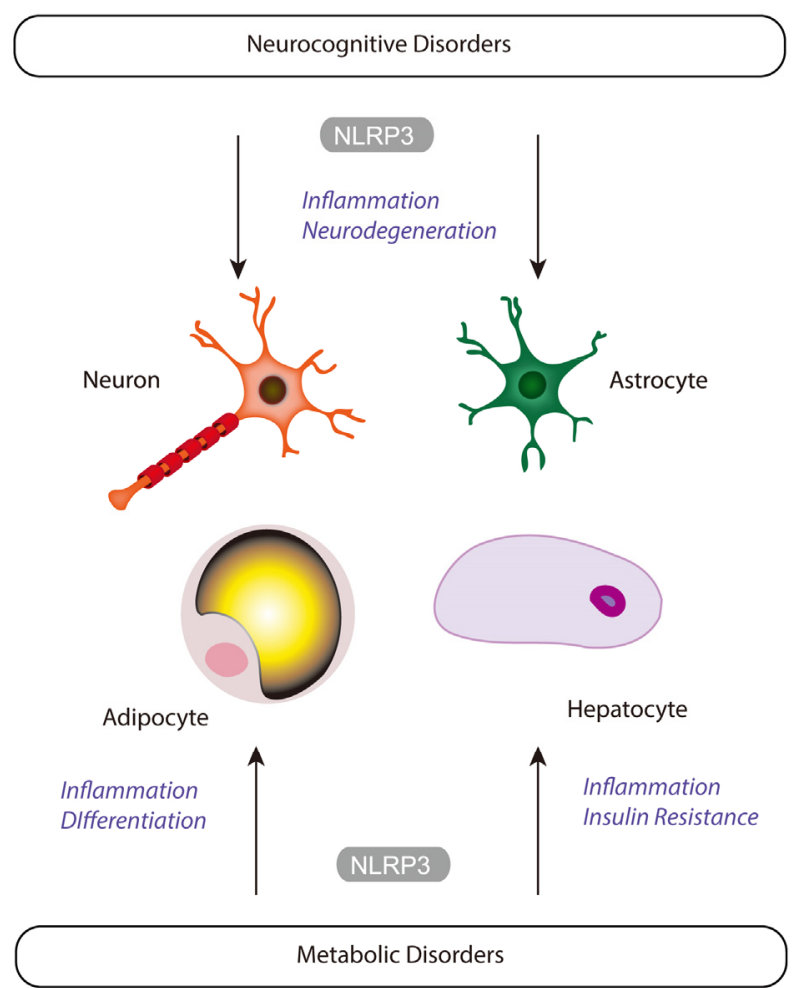

Fig. 2. NLRP3 has been implicated in pro-pathogenic responses related to several human diseases, including neurocognitive disorders and metabolic disorders, although the mechanisms remain incompletely clear. Neurons and astrocytes may represent targets of NLRP3-dependent inflammatory processes, leading to the promotion of neurocognitive disorders. Adipocyte differentiation and growth have been linked to NLRP3-dependent processes in obesity. Hepatic insulin resistance and inflammation may also be mediated in part by NLRP3-dependent inflammation.
ASC to interact directly with caspase-1. However, ASC has been shown to enhance NLRP1-mediated caspase-1 activation in vitro (Faustin et al., 2007). In contrast to most proteins of the NLR family, the NLRP3 protein lacks the typical CARD domain. The PYD domain of NLRP3 interacts with ASC, whose CARD domain recruits the CARD of pro-caspase-1 (Schroder and Tschopp, 2010). Finally, the IPAF protein lacks a PYD domain (Schroder and Tschopp, 2010), but contains a CARD domain that interacts directly with pro-caspase- 1 without the need of ASC (Poyet et al., 2001). Therefore, IPAF may interact with a PYD-containing protein (i.e., NLRPs) for appropriate inflammatory responses to invasive pathogens (Schroder and Tschopp, 2010).

\section{AlM2 inflammasome}

The pyrin and HIN domain-containing protein (PYHIN) family member absent in melanoma 2 (AIM2 inflammasome) represents the first non-NLR family member identified that can form an inflammasome complex (Ludlow et al., 2005; Schroder and Tschopp 2010). While most inflammasomes in the NLR family depend on a central NACHT domain for oligomerization, AIM2 uses multiple binding sites for cytosolic DNA via its C-terminal HIN200 domain. Similar to NLRP3, AIM2 contains a PYD domain that interacts with ASC via homotypic PYD-PYD interactions, which allows the CARD domain within ASC to regulate nuclear factor kappa B (NF-kB) and caspase-1 production (Hornung et al., 2009). As a result, AIM2-mediated caspase-1 activation induces pro-inflammatory cytokine secretion (i.e., IL1 $\beta$, IL-18) (Schroder and Tschopp, 2010).

\section{NLRP3 INFLAMMASOME ACTIVATION AND REGULATION}

\section{TLR-dependence}

TLRs are critical receptors that regulate inflammasome activity and pro-IL-1 $\beta$ production. TLR agonists, such as lipopolysaccharide (LPS), induce pro-IL-1 $\beta$ secretion via the NF- $\kappa B$ transcription factor and IL-1 $\beta$ promoter activation (Hiscott et al., 1993; Schroder and Tschopp, 2010). Prior to caspase-1-mediated pro- 
teolytic cleavage of IL-1 $\beta$, TLR agonists facilitate the NLRP3 inflammasome pathway through an NF-kB-dependent priming signal, which may represent a rate-limiting component of inflammasome complex formation (Bauernfeind et al., 2009; Guarda et al., 2011). Other studies have suggested the importance of TLR4 in the inflammasome pathway. For example, CD14, a phagocyte-expressed PRR known to interact with TLR4, increased TLR4-mediated inflammatory response to LPS (Fujihara et al., 2003) and monosodium urate (MSU) (Scott et al., 2006). Furthermore, LPS failed to induce NLRP3 or pro-IL-1 $\beta$ activation in cells lacking a functional TLR4 receptor (Bauernfeind et al., 2009). Franchi et al. (2009) demonstrated that the presence of ATP was required for TLR4-dependent caspase-1 activation following exposure to LPS.

\section{ATP-dependence}

Extracellular ATP activates the P2X purinoreceptor $7\left(\mathrm{P} 2 \mathrm{X}_{7} \mathrm{R}\right)$ via the pannexin-1 hemichannel, which was proposed to permit the bacterial ligand to enter the cytosol to induce caspase-1 expression (Kanneganti et al., 2007). P2X ${ }_{7}$ R-dependent procaspase-1 activation and IL-1 $\beta$ secretion have been found to facilitate NF-kB activation via a TNF- $\alpha$-dependent pathway, thereby suggesting an important role of $\mathrm{P}_{2} \mathrm{X}_{7} \mathrm{R}$ in NLRP3 inflammasome complex formation (Solini et al., 2013; Wen et al., 2011). ATP-mediated $P 2 X_{7} R$ stimulation has also been shown to induce a $\mathrm{K}^{+}$efflux, which indicates that reduced intracellular $\mathrm{K}^{+}$concentration triggers the NLRP3 inflammasome pathway (Pétrilli et al., 2007). Furthermore, suppressed NLRP3 activity has been reported to result from treatment with the ATPsensitive $\mathrm{K}^{+}$channel inhibitor, glibenclamide, and the chemical ROS scavenger, (2R,4R)-4-aminopyrrolidine-2, 4-dicarboxylate (APDC) (Allen, 2009; Nakahira, 2011). However, P2X 7 Rdeficient and wild type mice exhibit similar levels of adipocyte cell death in a diet-induced obesity model (Sun et al., 2012). Furthermore, a recent study has shown that pannexin-1 is not required for $\mathrm{P} 2 \mathrm{X}_{7} \mathrm{R}$ function during activation of the NLRP3, IPAF, and AIM2 inflammasomes (Qu et al., 2011). As a result, activation of the inflammasome pathway may not strictly depend on the presence of ATP in vivo.

\section{Alternative pathways and ROS-dependence}

Recent studies have proposed an alternative model for NLRP3 inflammasome formation that involves lysosomal destabilization due to engulfment of crystalline or particulate agonists (Schroder and Tschopp, 2010). For example, macrophage cells treated with silica crystals or MSU displayed swollen lysosomes and dispersion of lysosomal contents in the cytosol (Hornung et al., 2008). The antibiotic nigericin can induce lysosomal rupture via cathepsin $\mathrm{B}$, a cysteine protease found in the lysosome, and subsequent caspase- 1 activation via $\mathrm{K}^{+}$efflux (Heid et al. 2013; Hentze et al., 2003). Moreover, since pannexin-1 is required for nigericin-induced NLRP3 inflammasome formation (Pelegrin and Surprenant, 2006; 2007) and enables larger lysosomal pores, pannexin-1 may induce lysosomal destabilization (Hornung et al., 2008). Furthermore, the same study reported that cells undergoing lysosomal rupture lose cathepsin B, thereby inducing caspase-1 activation, which suggests that the NLRP3 inflammasome recognizes lysosomal damage and subsequent lysosomal content in the cytoplasm following phagocytosis of NLRP3 agonists (Hornung et al., 2008).

Environmental stimuli, such as asbestos and silica, can activate the NLRP3 inflammasome complex via phagocytic NADPH oxidase-induced reactive oxygen species (ROS) production (Dostert et al., 2008). For example, NADPH oxidase inhibitors, diphenylene iodonium (DPI) and apocynin, and knockdown of the NADPH oxidase subunit $\mathrm{p} 22^{\text {phox }}$ have been shown to reduce IL-1 $\beta$ production. Similar inhibitory effects on inflammasome activation have been reported for ROS inhibitors $\mathrm{N}$-acetyl-L-cysteine (NAC) and APDC, in response to asbestos, MSU, and ATP (Dostert et al., 2008). P2X 7 R has been shown to mediate NADPH oxidase-dependent oxidative stress both in vivo (Chatterjee et al., 2012) and in vitro (Korcok et al., 2004; Liu et al., 2011), thereby indicating a critical role for $P 2 X_{7} R$ in activation of the NLRP3 inflammasome via ROS signaling.

Furthermore, recent studies suggest that treatment of macrophages with the mitochondria-targeted antioxidant MitoTEMPO, a TEMPOL derivative that targets mitochondrial ROS (Trnka et al., 2009), reduced secretion of both IL-1 $\beta$ and IL-18. Mito-TEMPO inhibited subsequent caspase-1 activation and mitochondrial DNA (mtDNA) translocation to the cytosol in response to LPS and ATP (Nakahira et al., 2011). However, MitoTEMPO did not inhibit IL-1 $\beta$ secretion following exposure to treatment with LPS and MSU, indicating that this pathway is independent of mitochondrial ROS (Nakahira et al., 2011). In conclusion, while ROS production represents a critical prerequisite to the NLRP3 inflammasome pathway, the source of ROS may vary with experimental models.

\section{NLRP3 inflammasome in metabolic disorders}

Recently, the prevalence of metabolic disorders, such as obesity, type 2 diabetes mellitus (T2DM), and atherosclerosis has risen tremendously (Davis et al., 2011). The World Health Organization (WHO) estimates that over 2.8 million adults above the age of 20 die each year worldwide due to being overweight $\left(B M I \geq 25 \mathrm{~kg} / \mathrm{m}^{2}\right)$ or suffering from obesity $\left(B M I \geq 30 \mathrm{~kg} / \mathrm{m}^{2}\right)$. In $2008,10-14 \%$ of adults were clinically obese, in contrast to 5 $8 \%$ of men and women in 1980 (Finucane et al., 2011; WHO, 2011). Furthermore, obesity increases the risk of diabetes, heart disease, stroke, hypertension, and depression (WHO, 2002). In 2008, diabetes alone accounted for 1.3 million deaths (WHO, 2011). In recent years, several studies have illustrated the chronic pro-inflammatory processes involved in the onset of metabolic disorders, including obesity and diabetes (Davis et al., 2011).

Obesity can stem from a variety of factors, such as genetics, aging, and unhealthy lifestyle habits (Skeldon et al., 2014). Adipose tissue in obese individuals releases more free fatty acids, hormones (i.e., leptin), pro-inflammatory cytokines (i.e., TNF- $\alpha$, IL-1 $\beta$ ), signaling proteins, and transcription factors that lead to insulin resistance (Shoelson et al., 2006; Wellen and Hotamisligil, 2005). Insulin resistance associated with dysfunctional insulin release from pancreatic islet $\beta$-cells impairs blood glucose tolerance, which typifies the development of T2DM (Kahn et al., 2006).

\section{Obesity}

Inflammation is associated with metabolic disorders, such as obesity. Recent studies have shown that the NLRP3 inflammasome may contribute to obesity. For example, weight loss due to calorie restriction or exercise in obese individuals with T2DM not only reduced NLRP3 expression and inflammation in adipose tissue and liver, but also improved insulin sensitivity (Vandanmagsar et al., 2011). In addition, mice deficient in NLRP3, ASC, and caspase-1 did not exhibit obesity-induced insulin resistance, which suggests that the NLRP3 inflammasome may act as a cellular regulator of insulin sensitivity (Vandanmagsar et al., 2011). However, since loss of NLRP3 function did not affect caspase-1 activation in the kidney of 
obese mice, inflammasomes may contribute to obesity via tissue-specific mechanisms (Vandanmagsar et al., 2011).

Two recent reports have implicated that caspase-1 may regulate lipid metabolism, thereby controlling the induction of obesity. Both studies showed that Casp1 1 mice with lean phenotype exhibited reduced plasma triglyceride levels (Kotas et al., 2013; van Diepen et al., 2013). While van Diepen et al. (2013) illustrated a marked decrease in intestinal lipid absorption, Kotas et al. (2013) found enhanced triglyceride clearance from the circulation without alterations in lipid production or intestinal. The lipid clearance in Casp $1^{-1}$ mice was also observed in NLRP3 mice, irrespective of $\mathrm{IL}-1 \beta$ and $\mathrm{IL}-18$ signaling (Kotas et al., 2013). Therefore, the NLRP3 inflammasome appears to affect lipid metabolism in obesity independently of IL-1 $\beta$ and IL-18 signaling. However, negligible changes in triglyceride and cholesterol levels were observed in obese Casp $1^{-}$or $N L R P 3^{-}$mice (Stienestra et al., 2010; 2011; Vandanmagsar et al., 2011). Thus, the role of NLRP3 activation in lipid metabolism related to obesity remains unclear.

Release of the NLRP3 inflammasome following diet-induced obesity has been shown to induce adipocyte differentiation, which also enhanced IL-1 $\beta$ production and insulin resistance in adipose tissue (Stienstra et al., 2010) and increased adipocyte size (Stienstra et al., 2011). Elevated MSU levels have been linked to oxidative stress in adipocytes and obesity (Sautin et al., 2007). Considering the recognition of MSU by the NLRP3 inflammasome, thereby inducing an inflammatory response, NLRP3 activation may contribute to insulin resistance in obesity (Hornung et al., 2008; Martinon et al., 2006). Furthermore, inhibited recognition of such stimuli due to NLRP3 dysfunction may prevent chronic obesity (Vandanmagsar et al., 2011).

\section{Type 2 diabetes mellitus (T2DM)}

Type 2 diabetes mellitus (T2DM) is an auto-inflammatory disease induced by metabolic stressors, such as elevated levels of glucose and free fatty acids (FFAs) (Donath and Shoelson, 2011). Many studies have indicated a critical role of inflammatory processes in cases related to T2DM. For example, proinflammatory cytokines, TNF- $\alpha$, IL- $1 \beta$ and IFN $\gamma$, activate signaling pathways that result in pancreatic $\beta$-cell death and dysfunction (Eizirik et al., 2012; Feve et al., 2009; Mraz et al., 2011). As a result, elevated IL-1 $\beta$ levels and limited $\beta$-cell function are two primary predictors for the development of T2DM through inhibition of insulin signaling (Wang et al., 2014a). Additionally, recent studies have shown that inhibition of IL-1 $\beta$ production reduced T2DM prevalence in animal models (Emanuela et al., 2012; Mandrup-Poulsen et al., 2010). Furthermore, NLRP3dependent $\mathrm{IL}-1 \beta$ secretion during metabolic stress due to chronic hyperglycemia may contribute to the development of T2DM (Schroder et al., 2010). These findings suggest that IL$1 \beta$ production via NLRP3 inflammasome activation is involved in the pathogenesis of T2DM.

The NLRP3 inflammasome has been shown to bind to thioredoxin interacting protein (TXNIP), which facilitates ROSmediated NLRP3 activation via inhibition of thioredoxin (Skeldon et al., 2014). A recent study has illustrated a potential role for TXNIP in innate immunity via formation of the NLRP3 inflammasome and release of IL-1 $\beta$ in diabetes and oxidative stress (Devi et al., 2012). Also, pancreatic $\beta$-cell death and insulin resistance in T2DM have been associated with IL-1 $\beta$ secretion mediated by the ROS-TXNIP axis, thereby enhancing NLRP3 activation (Zhou et al., 2010). This involvement of ROSTXNIP in T2DM is consistent with the requirement for ROS in the NLRP3 inflammasome pathway (Wang et al., 2014a).
Therefore, the NLRP3 inflammasome may contribute to the pathogenesis of T2DM via the TXNIP protein (Wang et al., 2014a). However, the mechanisms that induce ROS generation in response to metabolic stress, such as chronic hyperglycemia remain incompletely understood.

Targeting specific components of the inflammasome pathway may provide a plausible approach for treatment of T2DM. For example, glibenclamide, a drug widely used to treat T2DM, has been shown to inhibit formation of the glucose-induced TXNIPNLRP3 inflammasome complex (Zhou et al., 2010). Palmitate, a saturated fatty acid found in plasma, triggers the secretion of IL-1 $\beta$ and IL-18 from macrophages via an NLRP3-dependent pathway (Wen et al., 2011). In addition, islet amyloid polypeptide (IAPP), a protein that forms amyloid deposits in the pancreas during T2DM, enhanced IL-1 $\beta$ secretion and NLRP3 activation in vitro (Masters et al., 2010). However, this same study reported that IL-1 $\beta$ release in response to IAPP depended on NLRP3, but not TXNIP (Masters et al., 2010). Therefore, TXNIP appears to represent an agonist-dependent protein within the NLRP3 inflammasome pathway.

\section{NLRP3 inflammasome in cognitive diseases}

Although neurocognitive diseases, such as Alzheimer's disease, Parkinson's disease, Huntington's disease, and major depressive disorder (MDD), are not often characterized as typical inflammatory diseases, recent research has indicated that activation of the innate immune system may contribute to their pathogenesis (Glass et al., 2010). PRRs, such as the TLR4 receptor, are highly expressed on immune cells (i.e., microglia and astrocytes) that induce inflammatory processes via cytokine secretion (Saijo et al., 2009). Considering that TLR4 polymorphisms have been linked to several human age-related diseases (i.e., Alzheimer's disease, atherosclerosis, and T2DM), such receptors may influence neurodegenerative diseases via inflammatory responses (Balistreri et al., 2009). In addition to PRRs, purinergic receptors (i.e., $P 2 X_{7} R$ ) are expressed on microglia and astrocytes. Furthermore, upregulation of this receptor has been reported in mouse models of Alzheimer's disease (Parvathenani et al., 2003). Thus, PRRs and purinergic receptors on immune cells appear not only to serve as initial sensors of microbial pathogens, but they also likely induce inflammatory pathways associated with cognitive diseases, such as Alzheimer's disease and MDD (Glass et al., 2010).

\section{Alzheimer's disease}

Alzheimer's disease is characterized by progressive dementia that stems from accumulation of amyloid-beta $(A \beta)$ plaques in the cerebrum, which leads to chronic inflammation and subsequent neuronal cell death (Leemans et al., 2011). During the 1980s, patients suffering from Alzheimer's disease exhibited elevated levels of microglia, the primary phagocyte in the central nervous system (CNS) (Itagaki et al., 1989; McGeer et al., 1987). Since then, amyloid plaques have been shown to activate microglia, thereby inducing the neuronal abnormalities associated with Alzheimer's disease (Meyer-Luehmann et al., 2008). However, it is still debated whether $A \beta$ accumulation is the result of chronic inflammation in amyloid-effected tissue or other disease-related processes (Leemans et al., 2011).

Numerous studies have identified a critical role for IL-1 $\beta$ and the NLRP3 inflammasome in the pathogenesis of Alzheimer's disease. For example, Halle et al. (2008) reported that $A \beta$ phagocytosis induced IL-1 $\beta$ secretion via NLRP3-dependent activation of caspase-1, thereby suggesting involvement of the NLRP3 inflammasome in Alzheimer's disease. Exposing LPS- 
primed macrophages to fibrillar $A \beta$ activated caspase- 1 and triggered IL-1 $\beta$ release; this response was dependent on NLRP3 and involved both endosomal rupture and cathepsin B release (Halle et al., 2008). Furthermore, inhibition of cathepsin $B$ has been shown to provide therapeutic benefit in a mouse model of Alzheimer's disease (Hook et al., 2008). Considering the role of cathepsin B in caspase-1 activation, this finding provides further evidence for the involvement of the NLRP3 inflammasome pathway in Alzheimer's disease.

A recent study reported upstream inflammatory processes in the context of Alzheimer's disease in vivo (Heneka et al., 2013). $N L R P^{-}$or Casp 1 mice crossed into APP/PS1 transgenic mice with mutations associated with Alzheimer's disease (Jankowsky et al., 2001) exhibited reduced symptoms associated with this cognitive disease, including chronic $A \beta$ secretion, neuronal inflammation, and cognitive impairment (Heneka et al., 2013). The same study demonstrated reduced release of caspase-1 and IL-1 $\beta$ in the brain and enhanced $A \beta$ clearance, which suggests that NLRP3 functions in the pathogenesis of Alzheimer's disease (Heneka et al., 2013). More importantly, these findings indicate that innate immune responses, specifically those related to inflammasome activation, contribute to neurocognitive disease onset and progression.

Major depressive disorder (MDD)

Major depressive disorder (MDD), also known as depression, is a severe neuropsychiatric illness that involves significant changes in mood, self-esteem, circadian rhythms, eating, and cognition (Iwata et al., 2013). While MDD affects at least $10 \%$ of the population in the United States (Kessler et al., 2003), its biosynthetic pathway remains unresolved. Previous studies suggest that environmental stimuli can lead to inflammatory processes (i.e., cytokine secretion and NLRP3 inflammasome activation), neurological changes, and MDD onset. For example, inflammatory cytokines (i.e., IL-1 $\beta$, IL-6, and TNF- $\alpha$ ) have been shown to play an important role in major depression (Dowlati et al., 2010; Maes et al., 1993). Moreover, Iwata and colleagues have proposed that IL-1 $\beta$ secretion represents the initial step of the pro-inflammatory response to psychological stress (Iwata et al., 2013). In agreement with this proposal, IL$1 \beta$ release has been implicated to facilitate depressive behaviors (i.e., anhedonia) via the NF-kB pathway following both acute (i.e., foot-shock or immobilization) and chronic unpredictable stress (Koo and Duman, 2008).

Recent literature has examined a close relationship between depression and the NLRP3 inflammasome. For example, patients diagnosed with MDD exhibit increased levels of cytokines, IL-1 $\beta$ and IL-18, as well as the NLRP3 inflammasome in peripheral blood mononuclear cells (PBMCs) (Alcocer-Gomez and Cordero, 2013). Also, IL-1 $\beta$ and NLRP3 inflammasome mRNA expression levels showed a significant increase in mouse brain following LPS-induced depression, which indicates that IL-1 $\beta$ and NLRP3 act as inflammatory mediators for psychological stress and depression (Zhang et al., 2013). In contrast to recent studies that suggest a critical role for mitochondrial rupture during NLRP3 activation (Latz et al., 2013; Nakahira et al., 2011), the inflammasome pathway in MDD patients does not depend on mitochondrial dysfunction (Alcocer-Gomez and Cordero, 2013). As a result, the mechanisms underlying the effect of psychological stress on inflammasome formation are still debated.

Of clinical relevance, several recent studies indicate that antidepressant medications regulate inflammasome activation. For example, amitriptyline, a common tricyclic antidepressant, has been shown to inhibit IL-1 $\beta$ and IL-18 production, as well as NLRP3 and caspase-1 gene expression in MDD patients (Alcocer-Gomez and Cordero, 2013). In addition, glyburide, an anti-diabetic drug that inhibits caspase- 1 activation and IL-1 $\beta$ secretion, may also serve as an effective inhibitor of NLRP3 formation following stress-induced depression (Lamkanfi et al., 2009). Furthermore, Warner-Schmidt and colleagues found an antagonistic relationship between antidepressant drugs and anti-inflammatory agents in patients with depression (WarnerSchmidt et al., 2011). Of note, selective serotonin reuptake inhibitors (SSRIs), but not tricyclic antidepressants, increased TNF- $\alpha$ and IFN $\gamma$ levels in the frontal cortex, which was inhibited by anti-inflammatory agents (i.e., NSAIDs) (Warner-Schmidt et al., 2011). This finding suggests that SSRIs and tricyclic antidepressants depend on different mechanisms to interact with antiinflammatory drugs. Therefore, future clinical trials should consider the different effects of anti-inflammatory drugs on the efficacy of antidepressant agents in patients suffering from depression (Warner-Schmidt et al., 2011).

Depression-induced NLRP3 activation has been shown to correlate with many comorbid systemic illnesses (i.e., diabetes, cancer, and cardiovascular disease) (Iwata et al., 2013; Menu and Vince, 2011). Since cytokine secretion and inflammasome formation respond to stimuli other than invasive pathogens (i.e. psychological stressors), the inflammasome may transduce such psychological stimuli into an inflammatory response. In an evolutionary sense, the inflammasome may have adapted its pathway over time to include psychological stress due to the social interaction and non-pathogenic danger signals from the environment (Raison and Miller, 2013). Considering the prevalence and potential consequences of major depressive disorder future research should continue to focus on the role of inflammasome activation in this disease.

\section{CONCLUSION}

Inflammasomes are multi-protein complexes involved in the immune system and inflammation. In this review, we have elaborated on the involvement of the NLRP3 inflammasome in various metabolic and cognitive diseases, including obesity, type 2 diabetes mellitus, Alzheimer's disease, and major depressive disorder. In addition, other neurodegenerative diseases, such as Parkinson's disease (Lu et al., 2014; Wang et al., 2014b) and Huntington's disease (Glinsky, 2008), have been associated with the NLRP3 inflammasome. While this review focused on NLRP3, other inflammasomes, such as those belonging to the NLR family and the AIM2 inflammasome, may provide a foundation for future research. Due to potential pleiotropic effects of inflammasome formation and their associated cytokines in various disease models, further elucidation of their regulation and functional significance is warranted. Novel therapeutic strategies for modulation of inflammasome activation should continue to be developed for possible clinical application in the near future.

\section{REFERENCES}

Alcocer-Gómez, E., de Miguel, M., Casas-Barquero, N., NúñezVasco, J., Sánchez-Alcazar, J.A., Fernández-Rodríguez, A., and Cordero, M.D. (2013). NLRP3 inflammasome is activated in mononuclear blood cells from patients with major depressive disorder. Brain Behav. Immun. 36, 111-117.

Allen, I.C., Scull, M.A., Moore, C.B., Holl, E.K., McElvania-TeKippe, E., Taxman, D.J., Guthrie E.H., Pickles, R.J., and Ting, J.P. (2009). The NLRP3 inflammasome mediates in vivo innate immunity to influenza A virus through recognition of viral RNA. Im- 
munity 30, 556-565.

Balistreri, C.R., Colonna-Romano, G., Lio, D., Candore, G., and Caruso, C. (2009). TLR4 polymorphisms and ageing: implications for the pathophysiology of age-related diseases. J. Clin. Immunol. 29, 406-415.

Bauernfeind, F.G., Horvath, G., Stutz, A., Alnemri, E.S., MacDonald, K., Speert, D., Fernandes-Alnemri, T., Wu, J., Monks, B.G., Fitzgerald, K.A., et al. (2009). Cutting edge: NF-kappaB activating pattern recognition and cytokine receptors license NLRP3 inflammasome activation by regulating NLRP3 expression. J. Immunol. 183, 787-791.

Chatterjee, S., Rana, R., Corbett, J., Kadiiska, M.B., Goldstein, J., and Mason, R.P. (2012). P2X7 receptor-NADPH oxidase axis mediates protein radical formation and Kupffer cell activation in carbon tetrachloride-mediated steatohepatitis in obese mice. Free Radic. Biol. Med. 52, 1666-1679.

Davis, B.K., Wen, H., and Ting, J.P. (2011). The Inflammasome NLRs in immunity, inflammation, and associated diseases. Ann. Rev. Immun 29, 707-735.

Devi, T.S., Lee, I., Hüttemann, M., Kumar, A., Nantwi, K.D., and Singh, L.P. (2012). TXNIP links innate host defense mechanisms to oxidative stress and inflammation in retinal Muller glia under chronic hyperglycemia: implications for diabetic retinopathy. Exp. Diabetes Res. 2012, 438238.

Donath, M.Y., and Shoelson, S.E. (2011). Type 2 diabetes as an inflammatory disease. Nat. Rev. Immunol. 11, 98-107.

Dostert, C., Pétrilli, V., Van Bruggen, R., Steele, C., Mossman, B.T. and Tschopp J. (2008). Innate immune activation through Nalp3 inflammasome sensing of asbestos and silica. Science 320, 674-677.

Dowlati, Y., Herrmann, N., Swardfager, W., Liu, H., Sham, L., Reim, E.K., and Lanctôt, K.L. (2010). A meta-analysis of cytokines in major depression. Biol. Psychiatry 67, 446-457

Eizirik, D.L., Sammeth, M, Bouckenooghe, T., Bottu, G., Sisino, G., Igoillo-Esteve, M., Ortis, F., Santin, I., Colli, M.L., Barthson, J., et al. (2012). The human pancreatic islet transcriptome: expression of candidate genes for type 1 diabetes and the impact of proinflammatory cytokines. PLoS Genet. 8, e1002552.

Emanuela, F., Grazia, M., Marco de, R., Maria Paola, L., Giorgio, F., and Marco, B. (2012). Inflammation as a link between obesity and metabolic syndrome. J. Nutr. Metab. 2012, 476380

Faustin, B., Lartigue, L., Bruey, J.M., Luciano, F., Sergienko, E., Bailly-Maitre, B., Volkmann, N., Hanein, D., Rouiller, I., and Reed, J.C. (2007). Reconstituted NALP1 inflammasome reveals two-step mechanism of caspase-1 activation. Mol. Cell 25, 713724

Feve, B., and Bastard, J.P. (2009). The role of interleukins in insulin resistance and type 2 diabetes mellitus. Nat. Rev. Endocrinol. 5, 305-311

Finucane, M.M., Stevens, G.A., Cowan, M.J., Danaei, G., Lin, J.K., Paciorek, C.J., Singh, G.M., Gutierrez, H.R. Lu, Y, Bahalim, A.N., et al. (2011). National, regional, and global trends in bodymass index since 1980: systematic analysis of health examination surveys and epidemiological studies with 960 country-years and 9.1 million participants. Lancet 337, 557-567.

Franchi, L., Eigenbrod, T., and Nuñez, G. (2009). Cutting edge: TNF-a mediates sensitization to ATP and silica via the NLRP3 inflammasome in the absence of microbial stimulation. J. Immunol. 183, 792-796.

Fujihara, M., Muroi, M., Tanamoto, K., Suzuki, T., Azuma, H., and Ikeda, H. (2003). Molecular mechanisms of macrophage activation and deactivation by lipopolysaccharide: roles of the receptor complex. Pharmacol. Ther. 100, 171-194

Glass, C.K., Saijo, K., Winner, B., Marchetto, M.C., and Gage, F.H. (2010). Mechanisms underlying inflammation in neurodegeneration. Cell 140,918-934

Glinsky, G.V. (2008). SNP-guided microRNA maps (MirMaps) of 16 common human disorders identify a clinically accessible therapy reversing transcriptional aberrations of nuclear import and inflammasome pathways. Cell Cycle 7, 3564-3576.

Guarda, G., Zenger, M., Yazdi, A.S., Schroder, K., Ferrero, I., Menu, P., Tardivel, A., Mattmann, C., and Tschopp, J. (2011). Differential expression of NLRP3 among hematopoietic cells. J. Immunol. 186, 2529-2534.

Halle, A., Hornung, V., Petzold, G.C., Stewart, C.R., Monks, B.G. Reinheckel, T., Fitzgerald, K.A., Latz, E., Moore, K.J., and Go- lenbock, D.T. (2008). The NALP3 inflammasome is involved in the innate immune response to amyloid-beta. Nat. Immunol. 9 , 857-865

Heid, M.E., Keyel, P.A., Kamga, C., Shiva, S., Watkins, S.C., and Salter, R.D. (2013). Mitochondrial reactive oxygen species induces NLRP3-dependent lysosomal damage and inflammasome activation. J. Immunol. 191, 5230-5238.

Heneka, M.T., Kummer, M.P., Stutz, A., Delekate, A., Schwartz, S. Vieira-Saecker, A., Griep, A., Axt, D., Remus, A., Tzeng, TC., et al. (2013). NLRP3 is activated in Alzheimer's disease and contributes to pathology in APP/PS1 mice. Nature 493, 674-678.

Hentze, H., Lin, X.Y., Choi, M.S., and Porter, A.G. (2003). Critical role for cathepsin $B$ in mediating caspase-1-dependent interleukin-18 maturation and caspase-1-independent necrosis triggered by the microbial toxin nigericin. Cell Death Differ. 10, 956968

Hiscott, J., Marois, J., Garoufalis, J., D'Addario, M., Roulston, A., Kwan, I., Pepin, N., Lacoste, J., Nguyen, H., Bensi, G., et al. (1993). Characterization of a functional NF-kappa B site in the human interleukin 1 beta promoter: evidence for a positive autoregulatory loop. Mol. Cell. Biol. 13, 6231-6240.

Hook, V.Y., Kindy, M., and Hook, G. (2008). Inhibitors of cathepsin B Improve memory and reduce b-amyloid in transgenic Alzheimer disease mice expressing the wild-type, but not the Swedish mutant, b-secretase site of the amyloid precursor protein. J. Biol. Chem. 283, 7745-7753

Hornung, V., Bauernfeind, F., Halle, A., Samstad, E.O., Kono, H. Rock, K.L., Fitzgerald, K.A., and Latz, E. (2008). Silica crystals and aluminum salts activate the NALP3 inflammasome through phagosomal destabilization. Nat. Immunol. 9, 847-856.

Hornung, V., Ablasser, A., Charrel-Dennis, M., Bauernfeind, F., Horvath, G., Caffrey, D.R., Latz, E., and Fitzgerald, K.A. (2009) AIM2 recognizes cytosolic dsDNA and forms a caspase-1activating inflammasome with ASC. Nature 458, 514-518.

Itagaki, S., McGeer, P.L., Akiyama, H., Zhu, S., and Selkoe, D. (1989). Relationship of microglia and astrocytes to amyloid deposits of Alzheimer disease. J. Neuroimmunol. 24, 173-182.

Iwata, M., Ota, K.T., and Duman, R.S. (2013). The inflammasome: pathways linking psychological stress, depression, and systemic illnesses. Brain Behav. Immun. 31, 105-114.

Jankowsky, J.L., Slunt, H.H., Ratovitski, T., Jenkins, N.A., Copeland N.G., and Borchelt, D.R. (2001). Co-expression of multiple transgenes in mouse CNS: a comparison of strategies. Biomol. Eng. 17, 157-165.

Kahn, S.E., Hull, R.L., and Utzschneider, K.M.. (2006). Mechanisms linking obesity to insulin resistance and type 2 diabetes. Nature $444,840-846$.

Kanneganti, T.D., Lamkanfi, M., Kim, Y.G., Chen, G., Park, J.H. Franchi, L., Vandenabeele, P., and Núñez, G. (2007). Pannexin1-mediated recognition of bacterial molecules activates the cryopyrin inflammasome independent of Toll-like receptor signaling. Immunity 26, 433-443.

Kessler, R.C., Berglund, P., Demler, O., Jin, R., Koretz, D., Merikangas, K.R., Rush, A.J., Walters, E.E., Wang, P.S., and National Comorbidity Survey Replication (2003). The epidemiology of major depressive disorder: results from the National Comorbidity Survey Replication (NCS-R). J. Am. Med. Assoc. 289, 3095-3105

Koo, J.W., and Duman, R.S. (2008). IL-1beta is an essential mediator of the antineurogenic and anhedonic effects of stress. Proc. Natl. Acad. Sci. USA 105, 751-756.

Korcok, J., Raimundo, LN. Ke HZ Sims, S.M and Dixon, S.J. (2004). Extracellular nucleotides act through P2X7 receptors to activate NF-kB in osteoclasts. J. Bone Miner. Res. 19, 642-651.

Kotas, M.E., Jurczak, M.J., Annicelli, C., Gillum, M.P., Cline, G.W., Shulman, G.I., and Medzhitov, R. (2013). Role of caspase-1 in regulation of triglyceride metabolism. Proc. Natl. Acad. Sci. USA $110,4810-4815$

Lamkanfi, M., Mueller, J.L., Vitari, A.C., Misaghi, S., Fedorova, A., Deshayes, K., Lee, W.P., Hoffman, H.M., and Dixit, V.M. (2009) Glyburide inhibits the Cryopyrin/Nalp3 inflammasome. J. Cell Biol. 187, 61-70.

Latz, E., Xiao, T.S., and Stutz, A. (2013). Activation and regulation of the inflammasomes. Nat. Rev. Immunol. 13, 397-411.

Leemans, J.C., Cassel, S.L., and Sutterwala, F.S. (2011). Sensing damage by the NLRP3 inflammasome. Immunol. Rev. 243, 152- 
162

Liu, Y., Xiao, Y., and Li, Z. (2011). P2X7 receptor positively regulates MyD88-dependent NF-kB activation. Cytokine 55, 229-236.

Lu, M., Sun, X.L., Qiao, C., Liu, Y., Ding, J.H., and Hu, G. (2014). Uncoupling protein 2 deficiency aggravates astrocytic endoplasmic reticulum stress and nod-like receptor protein 3 inflammasome activation. Neurobiol. Aging 35, 421-430.

Ludlow, L.E., Johnstone, R.W., and Clarke, C.J. (2005). The HIN200 family: more than interferon-inducible genes? Exp. Cell Res. 308, 1-17.

Maes, M., Bosmans, E., Meltzer, H.Y., Scharpé, S., and Suy, E. (1993). Interleukin-1 beta: a putative mediator of HPA axis hyperactivity in major depression? Am. J. Psychiatry 150, 1189-1193.

Mandrup-Poulsen, T., Pickersgill, L., and Donath, M.Y. (2010). Blockade of interleukin 1 in type 1 diabetes mellitus. Nat. Rev. Endocrinol. 6, 158-166.

Mariathasan, S., Newton, K., Monack, D.M., Vucic, D., French, D.M., Lee, W.P., Roose-Girma, M., Erickson, S., and Dixit, V.M. (2004) Differential activation of the inflammasome by caspase-1 adaptors ASC and Ipaf. Nature 430, 213-218.

Martinon, F., Burns, K., and Tschopp, J. (2002). The inflammasome: a molecular platform triggering activation of inflammatory caspases and processing of prolL-1 $\beta$. Mol. Cell 10, 417-426.

Martinon, F., Pétrilli, V., Mayor, A., Tardivel, A., and Tschopp, J. (2006). Gout-associated uric acid crystals activate the NALP3 inflammasome. Nature 440, 237-241.

Martinon, F., Mayor, A., and Tschopp, J. (2009). The inflammasomes: guardians of the body. Annu. Rev. Immunol. 27, 229-265.

Masters, S.L., Dunne, A., Subramanian, S.L., Hull, R.L., Tannahill, G.M., Sharp, F.A., Becker, C., Franchi, L., Yoshihara, E., Chen, Z., et al. (2010). Activation of the NLRP3 inflammasome by islet amyloid polypeptide provides a mechanism for enhanced IL-1b in type 2 diabetes. Nat. Immunol. 11, 897-904.

McGeer, P.L., Itagaki, S., Tago, H., and McGeer, E.G. (1987). Reactive microglia in patients with senile dementia of the Alzheimer type are positive for the histocompatibility glycoprotein HLADR. Neurosci. Lett. 79, 195-200.

Menu, P., and Vince, J.E. (2011). The NLRP3 inflammasome in health and disease: the good, the bad and the ugly. Clin. Exp. Immunol. 166, 1-15.

Meyer-Luehmann, M., Spires-Jones, T.L., Prada, C., Garcia-Alloza, M., de Calignon, A., Rozkalne, A., Koenigsknecht-Talboo, J., Holtzman, D.M., Bacskai, B.J., and Hyman, B.T. (2008). Rapid appearance and local toxicity of amyloid-beta plaques in a mouse model of Alzheimer's disease. Nature 451, 720-724.

Mraz, M., Lacinova, Z., Drapalova, J., Haluzikova, D., Horinek, A., Matoulek, M., Trachta, P., Kavalkova, P., Svacina, S., and Haluzik, M. (2011). The effect of verylow- calorie diet on mRNA expression of inflammation-related genes in subcutaneous adipose tissue and peripheral monocytes of obese patients with type 2 diabetes mellitus. J. Clin. Endocrinol. Metab. 96, E606-613.

Nakahira, K., Haspel, J.A., Rathinam, V.A., Lee, S.J., Dolinay, T., Lam, H.C., Englert, J.A., Rabinovitch, M., Cernadas, M., Kim, H.P., et al. (2011). Autophagy proteins regulate innate immune responses by inhibiting the release of mitochondrial DNA mediated by the NALP3 inflammasome. Nat. Immunol. 12, 222-230.

Parvathenani, L.K., Tertyshnikova, S., Greco, C.R., Roberts, S.B., Robertson, B., and Posmantur, R. (2003). P2X7 mediates superoxide production in primary microglia and is up-regulated in a transgenic mouse model of Alzheimer's disease. J. Biol. Chem. 278, 13309-13317.

Pelegrin, P., and Surprenant, A. (2006). Pannexin-1 mediates large pore formation and interleukin-1beta release by the ATP-gated P2X7 receptor. EMBO J. 25, 5071-5082.

Pelegrin, P., and Surprenant, A. (2007). Pannexin-1 couples to maitotoxin- and nigericin-induced interleukin-1beta release through a dye uptakeindependent pathway. J. Biol. Chem. 282, 2386-2394.

Pétrilli, V., Papin, S., Dostert, C., Mayor, A., Martinon, F., and Tschopp, J. (2007). Activation of the NALP3 inflammasome is triggered by low intracellular potassium concentration. Cell Death Differ. 14, 1583-1689.

Poyet, J.L., Srinivasula, S.M., Tnani, M., Razmara, M., FernandesAlnemri, T., and Alnemri, E.S. (2001). Identification of Ipaf, a human caspase-1-activating protein related to Apaf-1. J. Biol. Chem. 276, 28309-28313.
Qu, Y., Misaghi, S., Newton, K., Gilmour, L.L., Louie, S., Cupp, J.E., Dubyak, G.R., Hackos, D., and Dixit, V.M. (2006). Pannexin-1 is required for ATP release during apoptosis but not for inflammasome activation. J. Immunol. 186, 6553-6561.

Qu, Y., Misaghi, S., Newton, K., Gilmour, L.L., Louie, S., Cupp, J.E., Dubyak, G.R., Hackos, D., and Dixit, V.M. (2011). Pannexin-1 is required for ATP release during apoptosis but not for inflammasome activation. J. Immunol. 186, 6553-6561.

Raison, C.L., and Miller, A.H. (2013). Malaise, melancholia and madness: The evolutionary legacy of an inflammatory bias. Brain Behav. Immun. 13, 467-475.

Saijo, K., Winner, B., Carson, C.T., Collier, J.G., Boyer, L., Rosenfeld M.G., Gage, F.H., and Glass, C.K. (2009). A Nurr1/CoR EST pathway in microglia and astrocytes protects dopaminergic neurons from inflammation-induced death. Cell 137, 47-59.

Sautin, Y.Y., Nakagawa, T., Zharikov, S., and Johnson, R.J. (2007). Adverse effects of the classical antioxidant uric acid in adipocytes: NADPH oxidase-mediated oxidative/nitrosative stress. Am. J. Physiol. Cell Physiol. 293, C584-C596

Schroder, K., and Tschopp, J. (2010). The inflammasomes. Cell 140, 821-832.

Schroder, K., Zhou, R., and Tschopp, J. (2010). The NLRP3 inflammasome: a sensor for metabolic danger? Science 327, 296300

Scott, P., Ma, H., Viriyakosol, S., Terkeltaub, R., and Liu-Bryan, R. (2006). Engagement of CD14 mediates the inflammatory potential of monosodium urate crystals. J. Immunol. 177, 6370-6378.

Shoelson, S.E., Lee, J., and Goldfine, A.B. (2006). Inflammation and insulin resistance. J. Clin. Invest. 116, 1793-1801.

Skeldon, A.M., Faraj, M., and Saleh, M. (2014). Caspases and inflammasomes in metabolic inflammation. Immunol. Cell Biol. 92, 304-313.

Solini, A., Menini, S., Rossi, C., Ricci, C., Santini, E., Blasetti Fantauzzi, C., lacobini, C., and Pugliese, G. (2013). The purinergic $2 X 7$ receptor participates in renal inflammation and injury induced by high-fat diet: possible role of NLRP3 inflammasome activation. J. Pathol. 231, 342-353.

Stienestra, R., Joosten, L.A., Koenen, T., van Tits, B., van Diepen, J.A., van den Berg, S.A., Rensen, P.C., Voshol, P.J., Fantuzzi, G., Hijmans, A., et al. (2010). The inflammasome-mediated caspase- 1 activation controls adipocyte differentiation and insulin sensitivity. Metabolism 12, 593-605.

Stienestra, R., van Diepen, J.A., Tack, C.J., Zaki, M.H., van de Veerdonk, F.L., Perera, D., Neale, G.A., Hooiveld, G.J., Hijmans, A., Vroegrijk, I., et al. (2011). Inflammasome is a central player in the induction of obesity and insulin resistance. Proc. Natl. Acad. Sci. USA 108, 15324-15329.

Sun, S., Xia, S., Ji, Y., Kersten, S., and Qi, L. (2012). The ATP-P2 x 7 signaling axis is dispensable for obesity-associated inflammasome activation in adipose tissue. Diabetes 61, 1471-1478.

Trnka, J., Blaikie, F.H., Logan, A., Smith, R.A., and Murphy, M.P. (2009). Antioxidant properties of MitoTEMPOL and its hydroxylamine. Free Radic Res. 43, 4-12.

Vandanmagsar, B., Youm, Y.H., Ravussin, A., Galgani, J.E., Stadler, K., Mynatt, R.L., Ravussin, E., Stephens, J.M., and Dixit, V.D. (2011). The NLRP3 inflammasome instigates obesity-induced inflammation and insulin resistance. Nat. Med. 17, 179-188.

van Diepen, J.A., Stienstra, R., Vroegrijk, I.O., van den Berg, S.A., Salvatori, D., Hooiveld, G.J., Kersten, S., Tack, C.J., Netea, M.G., Smit, J.W., et al. (2013). Caspase-1 deficiency in mice reduces intestinal triglyceride absorption and hepatic triglyceride secretion. J. Lipid Res. 54, 448-456.

Wang, H., Capell, W., Yoon, J.H., Faubel, S., and Eckel, R.H. (2014a). Obesity development in caspase-1-deficient mice. Int. J. Obes. (Lond) 38, 152-155.

Wang, L., Zhai, Y.Q., Xu, L.L., Qiao, C., Sun, X.L., Ding, J.H., Lu, M. and Hu, G. (2014b). Metabolic inflammation exacerbates dopaminergic neuronal degeneration in response to acute MPTP challenge in type 2 diabetes mice. Exp. Neurol. 251, 22-29.

Warner-Schmidt, J.L., Vanover, K.E., Chen, E.Y., Marshall, J.J., and Greengard, P. (2011). Antidepressant effects of selective serotonin reuptake inhibitors (SSRIs) are attenuated by antiinflammatory drugs in mice and humans. Proc. Natl. Acad. Sci. USA 108, 9262-9267.

Wellen, K.E., and Hotamisligil, G.S. (2005). Inflammation, stress, and diabetes. J. Clin. Invest. 115, 1111-1119. 
Wen, H., Gris, D., Lei, Y., Jha, S., Zhang, L., Huang, M.T., Brickey, W.J., and Ting, J.P. (2011). Fatty acid-induced NLRP3-ASC inflammasome activation interferes with insulin signaling. Nat. Immunol. 12, 408-415.

World Health Organization. (2002). The world health report 2002 Reducing risks, promoting healthy life. Geneva WHO 1-167.

World Health Organization. (2011). Global status report on noncommunicable diseases 2010. Geneva WHO 1-162.
Zhang, Y., Liu, L., Peng, Y.L., Liu, Y.Z., Wu, T.Y., Shen, X.L., Zhou, J.R., Sun, D.Y., Huang, A.J., Wang, X., et al. (2013). Involvement of inflammasome activation in lipopolysaccharide-induced mice depressive-like behaviors. CNS Neurosci. Ther. 20, 119124

Zhou, R., Tardivel, A., Thorens, B., Choi, I., and Tschopp, J. (2010) Thioredoxin-interacting protein links oxidative stress to inflammasome activation. Nat. Immunol. 11, 136-140. 